\title{
Les Calanques : territoire de science et source d'inspiration - Retour d'expérience sur la collaboration artiste-chercheur
}

\section{The Calanques: a land of science and source of inspiration - Feedback on artist-researcher collaboration}

\author{
Shanta Rao ${ }^{1}$, Justine Gadreaud ${ }^{2}$, Guillaume Marchessaux ${ }^{3,4}$ \\ ${ }^{1}$ Artist, Paris, France, contact@shantarao.net \\ ${ }^{2}$ Aix Marseille Univ, Univ Avignon, CNRS, IRD, IMBE, Marseille, France, justine.gadreaud@gmail.com \\ ${ }^{3}$ Aix Marseille Univ, Univ Toulon, CNRS, IRD, MIO, Marseille, France, guillaume.gmarchessaux@gmail.com \\ ${ }^{4}$ Université de Franche-Comté - Laboratoire Chrono-Environnement, UMR CNRS/UFC6249, F-25211 Montbéliard \\ cedex, France.
}

RÉSUMÉ. Lors de la résidence Les Calanques, territoire de science et source d'inspiration, l'artiste plasticienne Shanta Rao a exploré l'univers des méduses dont la présence grandissante sur les plages du Parc national des Calanques (région de Marseille, sud de la France) repose la question de l'altérité, des liens entre l'humain et les autres espèces animales. Expérience collaborative avec les chercheurs en biologie marine Justine Gadreaud et Guillaume Marchessaux (Aix-Marseille Université), les recherches de l'artiste autour du transformisme biologique spécifique à certaines méduses ont donné lieu à des œuvres à la croisée de la peinture et de la sculpture ainsi que des images photographiques et vidéographiques mettant en parallèle dispositifs de recherche plastique et scientifique. Suite à une première exposition de fin de résidence au Fonds Régional d'Art Contemporain Provence-Alpes-Côte-d'Azur à Marseille (2018), le projet initial a continué de se déployer par la réalisation de nouvelles œuvres et leur exposition notamment à la galerie institutionnelle Edouard Manet (Gennevilliers/FR, 2019), à la galerie Joseph Tang (Paris/FR, 2019), à l'espace d'art Shimmer (Rotterdam/NL, 2019) et au centre d'art contemporain Nest (La Haye/NL, 2020).

ABSTRACT. During the residency The Calanques, a land of science and source of inspiration, the visual artist Shanta Rao explored the world of jellyfish, whose growing presence on the beaches of the Parc national des Calanques (Marseille region, southern France) raises the question of otherness, of the links between humans and other animal species. A collaborative experiment with marine biology researchers Justine Gadreaud and Guillaume Marchessaux (AixMarseille University), the artist's research into the biological transformation specific to certain jellyfish has given rise to works at the crossroads of painting and sculpture, as well as photographic and video images that bring together plastic and scientific research devices. Following a first exhibition at the end of the residency at the Fonds Régional d'Art Contemporain Provence-Alpes-Côte-d'Azur in Marseille (2018), the initial project continued to develop through the creation of new works and their exhibition, notably at the Edouard Manet institutional gallery (Gennevilliers/FR, 2019), the Joseph Tang gallery (Paris/FR, 2019), the Shimmer art space (Rotterdam/NL, 2019) and the Nest contemporary art centre (The Hague/NL, 2020).

MOTS-CLÉS. Shanta Rao, Fondation Camargo, Méduses, Artiste en résidence, relation art et science, OSU Pythéas, Parc national des Calanques.

KEYWORDS. Shanta Rao, Camargo Foundation, Jellyfish, Artist in residence, relation between art and science, OSU Pytheas, National Park of Calanques.

\section{Une rencontre transdisciplinaire entre artiste et scientifiques}

\section{Les résidences de recherche et création, un lieu d'échange}

Les résidences offrent le temps, les moyens et l'espace, dans un environnement privilégié, pour penser, créer et échanger. Certaines d'entre elles encouragent les approches interdisciplinaires en favorisant les liens entre recherche scientifique et création artistique. 
En janvier 2018, le Parc national des Calanques, l'OSU Institut Pythéas qui regroupe plus de 1200 chercheurs autour des grandes thématiques des sciences de la Terre, de l'Environnement et de l'Univers et la fondation Camargo, fondation américaine qui, depuis presque cinquante ans, accueille sur son lieu de résidence à Cassis dans le sud de la France artistes et chercheurs du monde entier, ont invité huit artistes internationaux à réfléchir aux liens Humain/Nature dans le contexte exemplaire de l'unique parc national urbain d'Europe, le Parc national des Calanques. Durant cinq semaines, huit visions singulières se sont nourries d'échanges avec le territoire et ceux qui le vivent - chercheurs, agents du parc, habitants - et ont ébauché réflexions et propositions plastiques restituées lors d'une exposition au Fonds Régional d'Art Contemporain Provence-Alpes-Côte-d'Azur (Marseille, France), d'interventions publiques et d'une publication de la revue SEMAINE (revue hebdomadaire pour l'art contemporain, édition papier).

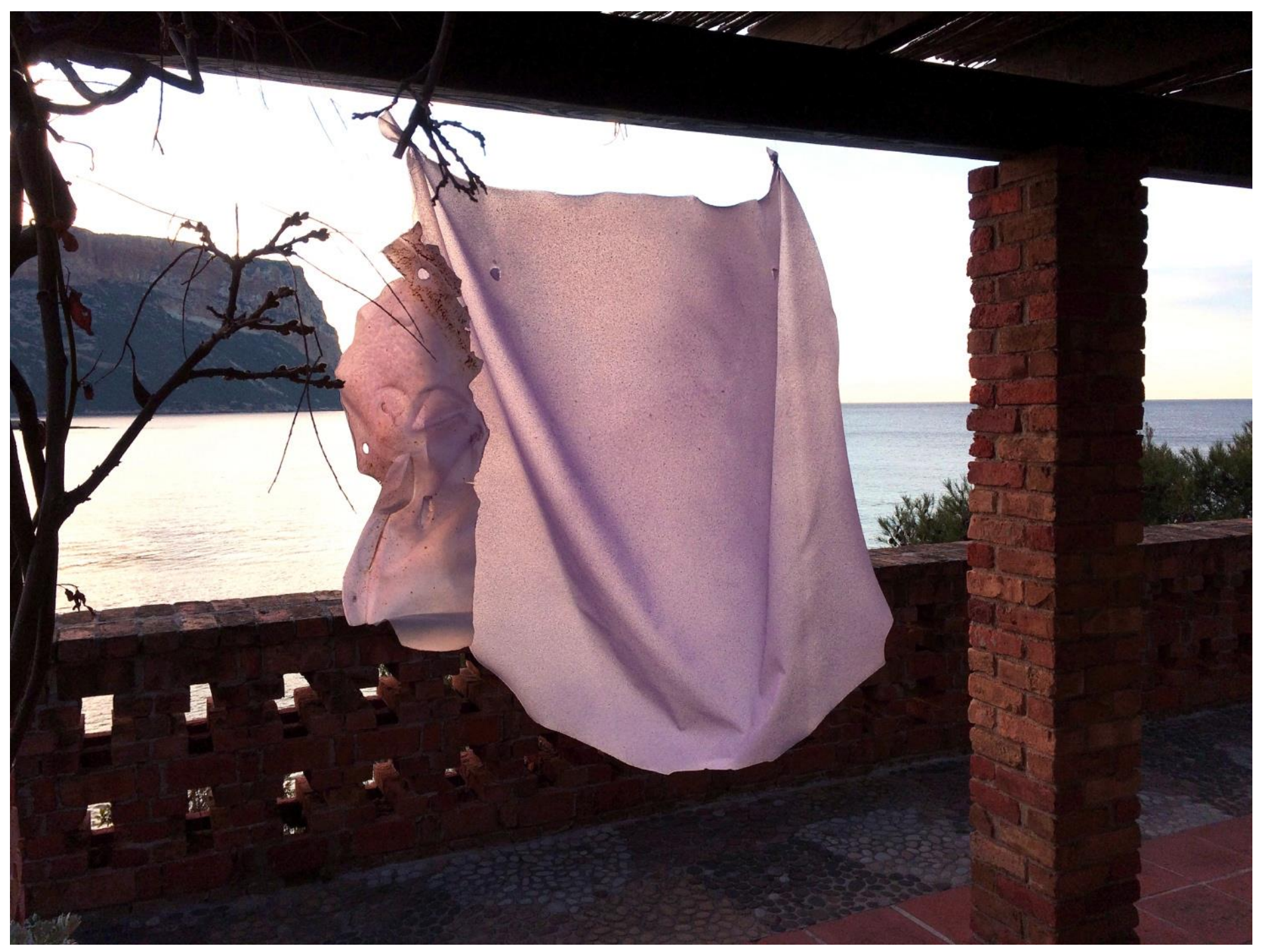

[1] Shanta Rao, work-in-progress, journée portes ouvertes à la Fondation Camargo, 2018

Lors de cette résidence, l'artiste Shanta Rao et les chercheurs Justine Gadreaud et Guillaume Marchessaux ont pu échanger, via leur intérêt pour les organismes gélatineux et plus particulièrement les méduses, autour des questions de morphogenèse et métamorphisme, de matière molle ou fluides complexes, de ductilité et mutabilité des formes, thématiques communes à l'art et à la science.

Dans le contexte spécifique de cette résidence, les échanges entre l'artiste et les chercheurs, tout autant discursifs qu'intuitifs, ont été rendus possibles par la sensibilisation aux champs d'investigation respectifs selon différentes approches, iconographique, textuelle et haptique lors de rencontres au sein de laboratoires de recherche ou sur le site de la fondation Camargo, aux abords des eaux marines du Parc national des Calanques. 


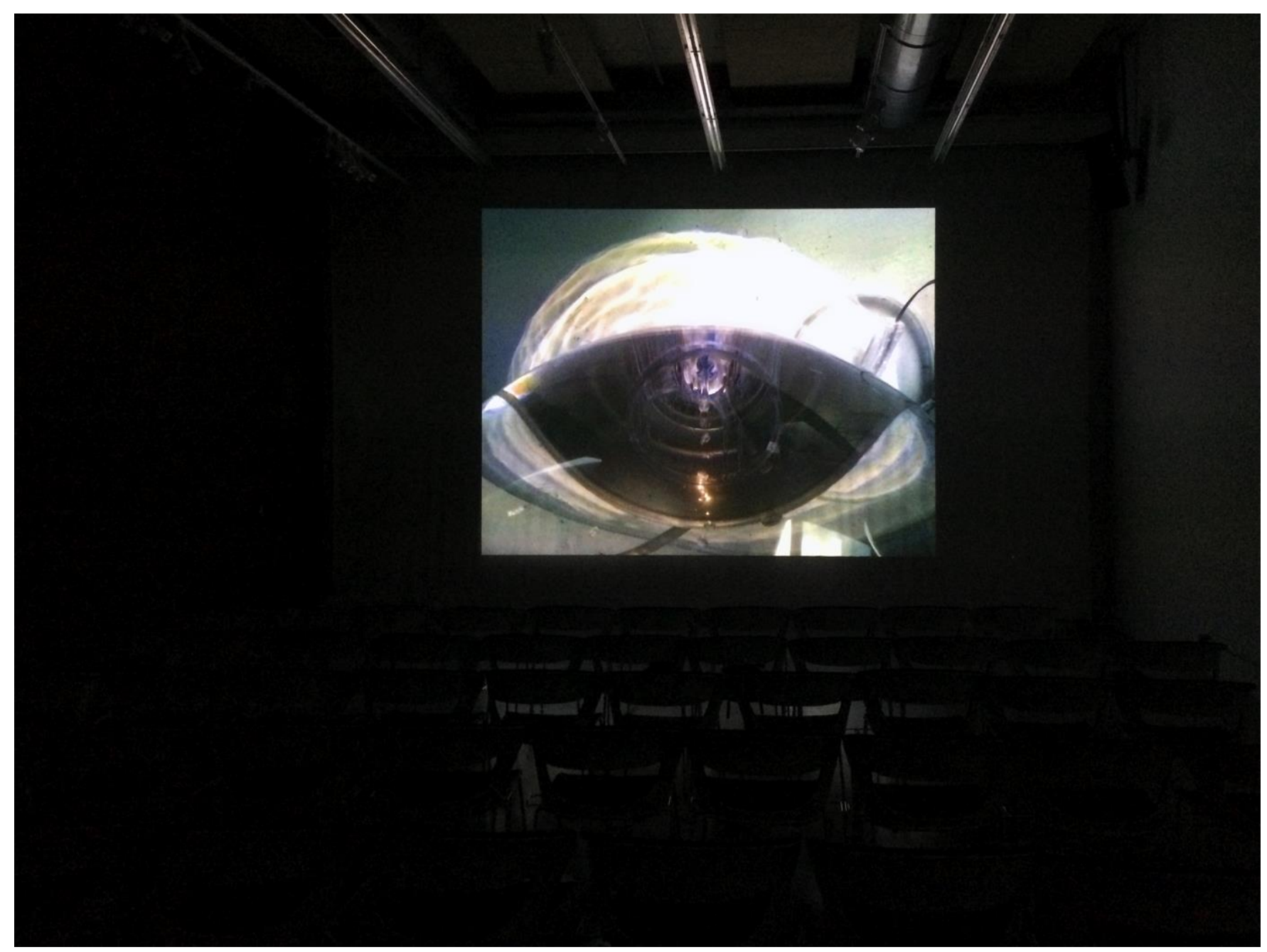

[2] Vue de la projection et intervention publique de Shanta Rao et Guillaume Marchessaux dans le cadre de l'exposition Les Calanques, territoire de science et source d'inspiration. Fond Régional d'Art Contemporain Provence-Alpes-Côte-d'Azur, Marseille, 2018

\section{Présentations}

- Shanta Rao - Des fréquences hertziennes se transforment en matière molle; des aplats d'encre phagocytent des images matricielles ; des compositions musicales perforent des lames siliconées ; des dessins numériques se dilatent, se météorisent; des gestes se déploient en diagrammes puis en paysages électroniques ; des textes littéraires se magnétisent ; des photographies se délitent en grains, en taches et se coagulent sur des surfaces caoutchouteuses; des pixels embossent des plaques de cuivre ; des figures géométriques mutent en partitions musicales impossibles à jouer.

L'ensemble de mes œuvres invoque un monde transformiste, en perpétuelle mutation. Elles puisent leurs origines dans le concret de phénomènes physiques et biologiques, ou dans l'abstraction d'algorithmes empruntés à la musique contemporaine, ou encore à l'informatique. Par une approche brouillant ou invalidant les généalogies, des objets-sources mutent, s'hybrident et réapparaissent dans un autre champ que celui d'origine, sous de nouvelles formes. Changement de langage, de forme, de matière, ce sont des œuvres-transfuges, une mise en forme de l'entropie. Le continuum transformatif des œuvres ne s'arrête pas à leur réalisation, mais se prolonge également lors de leur exposition, où elles empruntent des présentations variables, évoluant selon les contextes et les interactions avec les commissaires d'exposition. Certaines d'entre elles poursuivent même une évolution post-exposition, par de nouvelles transformations. Selon cette approche métamorphe, les œuvres peuvent s'incarner dans une grande variété de manifestations plastiques, autonomes ou s'intégrant dans des installations. Les plus récentes de ces manifestations, des sculptures exclusivement constituées de peinture, 
appartiennent au vaste champ de la soft sculpture, et ses problématiques visuelle, matérielle et temporelle.

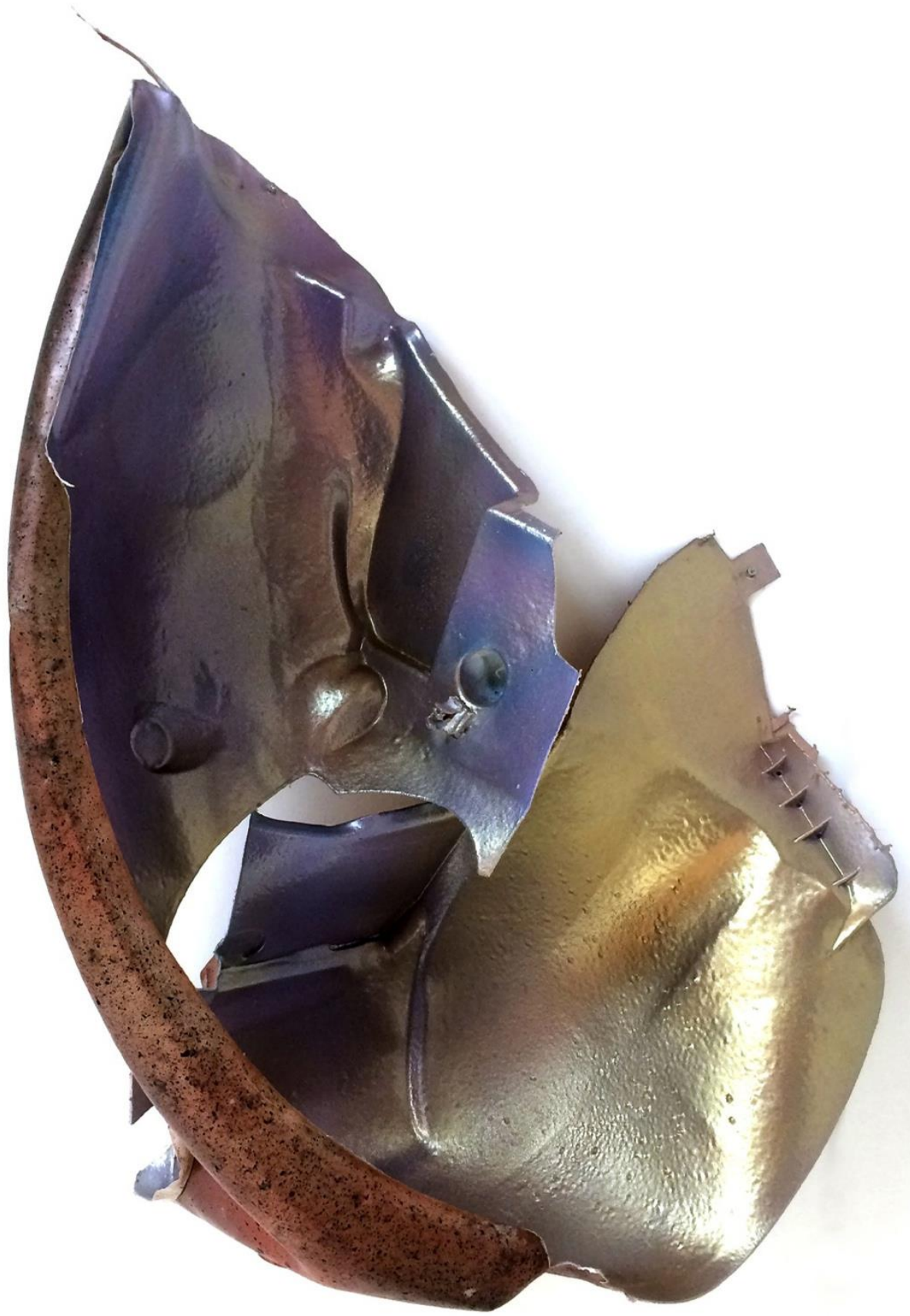

[3] Shanta Rao, Sans titre, 2019, peinture polymère, $98 \times 67 \times 33 \mathrm{~cm}$

- Guillaume Marchessaux - La recherche académique m'a toujours passionné. Au cours de mon parcours universitaire je me suis particulièrement intéressé à l'étude du plancton, petits organismes errants au gré des courants. Au sein de ce compartiment planctonique très diversifié, allant des cellules phytoplanctoniques aux crustacés, je me suis passionné par le zooplancton gélatineux. Ces organismes d'apparence très primitive sont en réalité fascinant d'ingéniosité et d'adaptations. Au cours de mon doctorat, j'ai travaillé sur une espèce de cténophore invasive, Mnemiopsis leidyi, dans les étangs de Berre et de Camargue, dans le Sud de la France. Cette invasion de gélatineux provoque d'importants impacts socio-écologiques allant de la perturbation du bon fonctionnement de l'écosystème lagunaire en passant par une importante gêne pour la pêche artisanale et la baignade. Mon travail consistait à quantifier ces impacts écologiques et sociologiques dans le but de proposer des mesures de gestion. 
Aujourd'hui chercheur, je travaille sur l'invasion d'une méduse d'eau douce, Craspedacusta sowerbii, originaire de Chine, proliférant dans les lacs, rivières et bassins continentaux. Dans le cadre de cette étude, mes recherches sont axées sur le cycle de reproduction de cette méduse fascinante et sur sa distribution en France métropolitaine.

- Justine Gadreaud - Passionnée par la vie sous-marine depuis l'enfance, ma scolarité puis mon parcours académique m'ont menée jusqu'au doctorat. Au-delà de cette fascination pour ces espaces sous-marins - semblant infini - et cette variété d'organismes, comprendre l'impact des activités humaines sur le fonctionnement de ces écosystèmes m'a toujours intéressée. La discipline scientifique de l'écotoxicité s'est alors imposée à moi : les études en écotoxicologie ont pour objectif de déterminer, de quantifier et de prédire les impacts que peuvent avoir des xénobiotiques sur les organismes vivants et leurs écosystèmes. Elles s'inscrivent plus largement dans une volonté politique de prévention des risques pour la sauvegarde de l'environnement et une problématique de santé publique. La découverte du modèle méduse s'est faite dans ce cadre. Ma recherche consiste à tester l'impact de nanoparticules manufacturées (argent et titane) sur le développement de la méduse Aurelia aurita via des expériences en laboratoire d'expositions. C'est dans ce cadre que mes connaissances sur la biologie de ces organismes gélatineux n'ont cessées de croître, et ma fascination avec : la fragilité de leur forme (98\% d'eau) associée à leur résistance physiologique, leur capacité d'adaptation et la résilience de leur population, leur cycle de vie et des méthodes de reproductions si particulières.

\section{Dialogue artiste-chercheur : De la biologie aux arts plastiques, protocoles et détournements}

- Shanta Rao - A l'origine, notamment lors d'une première résidence à la fondation Camargo, mon intérêt pour les méduses était purement théorique, j’y voyais un outil de réflexion complétant ma perspective artistique, mon intérêt pour l'évolution et la mutabilité des formes. La première méduse qui a attisé ma curiosité est la Turritopsis nutricula, exemplaire par sa capacité à changer de forme durant son existence jusqu'à revenir parfois au stade primaire de cellules indifférenciées, informe en quelque sorte ou plutôt protoforme. Lors de mes visites dans les laboratoires auxquels vous étiez rattachés, j'ai pu entrer en relation avec "vos méduses », parfois jusqu'à l'haptique, et découvrir ces organismes remarquables. Mais comment appréhender ces êtres gélatineux et plus généralement un environnement fluide où nos schémas perceptifs liés au monde terrestre, à la pesanteur, à une dimensionnalité réduite - nous ne voyons pas en dessous de nous - sont souvent inopérants? Quels sont les outils et méthodes que vous utilisez pour regarder, étudier ce milieu spécifique qui trouble nos représentations?

- Justine Gadreaud - Mon appréhension de notre environnement, son observation et la compréhension des mécanismes qui le régissent se fait par la science. La science regroupe un ensemble de disciplines qui utilisent un catalogue de méthodes ; méthodes qui ont fait preuve de leur efficacité pour décrire et comprendre le monde dans lequel l'humain évolue. Les expérimentations, les statistiques, la modélisation, etc. sont autant d'outils de la démarche scientifique qui servent à tester des hypothèses. Les résultats obtenus sont comparés, discutés et retranscrits dans des articles revus et validés par les pairs. La science est donc une discipline qui ne cesse de s'incrémenter de nouveaux outils, et dont les résultats - les connaissances - sont constamment remises en question. Mon travail de recherche regroupe plusieurs disciplines scientifiques mais complémentaires : chimie, aquariologie, biologie moléculaire et imagerie entres autres. Chacune de ces disciplines possèdent ses propres outils - le matériel (éprouvette, aquarium, pipette, outils de mesure, etc.) - et leurs propres méthodes (plans d'expérimentation, statistiques, modèle, etc.) pour tester des hypothèses. Bien sûr notre appréhension « du monde » par la science est limitée par nos moyens techniques et technologiques de mesure et d'analyse. Selon moi, ce sont des limites dont nous sommes totalement affranchies dans l'expérience de pensée artistique.

- Guillaume Marchessaux - Une des problématiques préliminaires pour aborder ce champ d'investigation commun est la nécessité de transposition, translation de notre lecture terrestre à la 
physis du milieu marin. L'océan est un vaste milieu, le plus vaste, il faut rappeler que la planète Terre est recouverte de $71 \%$ d'eau. L'océan s'étend à perte de vue, il est imprévisible, presque inaccessible, étendu sans limite. L'océan est un monde en trois dimensions. Il comprend en son horizontale les mouvements des masses d'eau, créés par les courants de surface mais aussi de fond grâce aux différentiels de températures et/ou de salinité : c'est la circulation thermohaline. Sur la verticale, l'océan se découpe de plusieurs étagements disposés en feuillets entre le fond et la surface, chacun englobant des caractéristiques chimiques, physiques et biologiques qui leur sont propres. Ces caractéristiques vont conditionner le développement de la vie et la composition taxonomique.

- Shanta Rao - Cuboméduse, cténaire ailé, méduses à l'envers, déesse des eaux saumâtres, corps de feu, piqueuse mauve, crinières de lion, méduse soucoupe volante, galère portugaise, ceintures de vénus, méduse immortelle, prismes flottants, ce champ du vivant est à lui seul une histoire des formes et le métamorphisme de certaines de ces méduses, qui ont longtemps esquivé l'ordination taxonomique, pose question. Je ne peux m'empêcher ici d'évoquer le paradoxe philosophique du bateau de Thésée et ses questions d'identité(s) à travers le temps qui sous-tend l'ensemble de mes recherches. Selon la légende, lorsque Thésée revint à Athènes après sa victoire contre le Minotaure, les Athéniens conservèrent son bateau. Mais avec le temps, afin de conserver le symbole de leur victoire à travers les siècles, ils durent remplacer les planches endommagées une par une au point qu'il ne restait plus de planche d'origine. S'agit-il toujours du bateau de Thésée ou d'un autre bateau ? L'identité persiste-elle par la forme, par la matière ou encore autrement ? Le paradoxe du bateau de Thésée ouvre sur la question du Même et de l'Autre, de la continuité spatio-temporelle des êtres et des choses. Mais que dire de ces méduses qui remettent en cause la linéarité du temps biologique et son unidirectionnalité, capables d'une évolution "à rebours », rajeunissant, se clonant ou encore s'incarnant dans des formes si diverses? Comment ces organismes dits primitifs ont-ils la possibilité génétique de revêtir différentes enveloppes charnelles, nous qui n'en avons qu'une? Comment la science explique-t-elle ces phénomènes?

- Guillaume Marchessaux - Les méduses et autres organismes gélatineux peuvent être considérés comme primitifs quand on sait qu'ils sont apparus il y a plus de 600 millions d'années. Il existe des milliers d'espèces d'organismes gélatineux allant des cnidaires, en passant par les cténaires ou les tuniciers. Les méduses sont composées d'un derme constitué principalement de collagène et de cellules musculaires striées très puissantes assurant la propulsion. Les méduses n'ont pas d'organes à proprement parler (i.e. poumons, cœur, cerveau, etc.). Elles sont composées d'un réseau complexe de cellules nerveuses et de fibres assurant les fonctions vitales (propulsion, extension des tentacules et filaments, etc.). Les organismes gélatineux respirent sans poumons, mais grâce à des échanges gazeux entre les cellules du derme et l'eau. Les principaux organes que l'on observe chez les méduses sont les gonades et l'estomac, le tout relié à une bouche. Cette bouche dont l'accès est directement relié à la cavité gastrique se trouve dans le prolongement des tentacules ou bras buccaux. Ces derniers pouvant se dilater jusqu'à 10 fois leur taille sont tapissés de cellules urticantes. Ces cellules remplies de poison permettent de capturer les proies qui seront ramenées à la bouche grâce aux mouvements contractiles des bras buccaux. Les proies sont digérées dans la cavité gastrique et le broyat est directement distribué à l'ensemble de l'organisme à travers le vaste réseau de canaux radiaires.

Chez les méduses, le cycle de vie est composé de deux stades : une reproduction sexuée pélagique et une reproduction asexuée benthique. Les phases méduses pélagiques sont sexuées. Les mâles et les femelles vont généralement rejeter leurs gamètes dans le milieu environnant. Après fécondation, une larve ciliée appelée planula se développe et plonge vers les profondeurs pour se fixer sur des roches, coquilles de bivalves, etc. Le développement de cette larve donnera lieu en un polype constitué d'un pied et de plusieurs dizaines de tentacules. Les polypes sont sexués, il y a donc des mâles et des femelles. Mais ils vont se reproduire par bourgeonnement asexué, chaque individu est donc un clone, donnant lieu à d'immenses colonies. Ces polypes vont donner ensuite naissance par strobilation ou bourgeonnement à de nouvelles larves de méduses pélagiques. Le cycle est ainsi bouclé. 


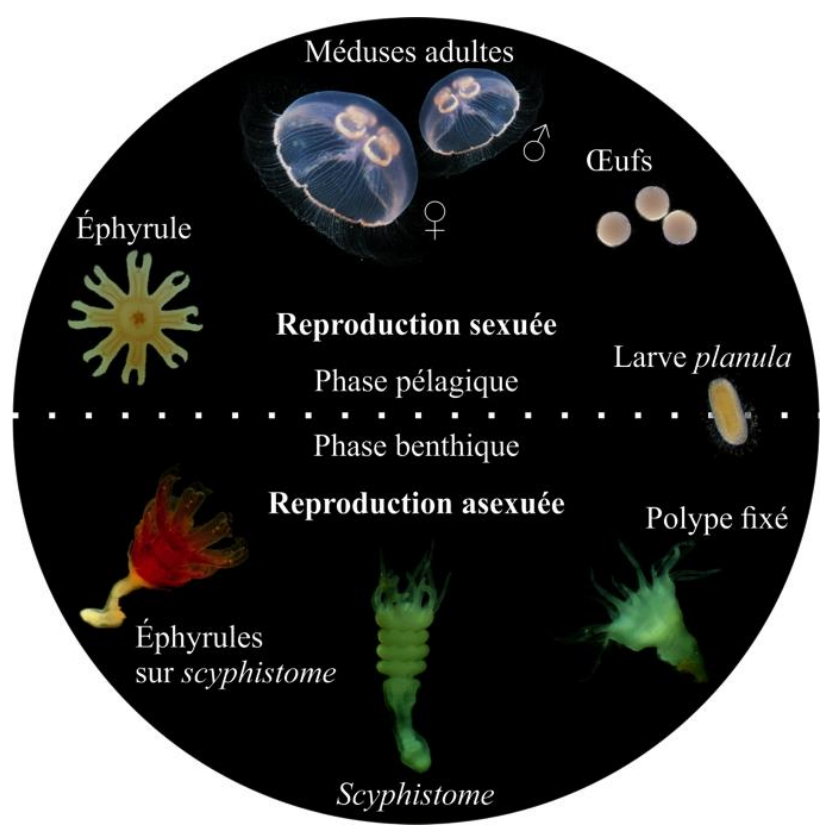

[4] Cycle de vie de la méduse Aurelia aurita.

Il existe une multitude de cycles de reproductions chez les autres organismes gélatineux. Certains comme les cténaires sont hermaphrodites et possèdent un cycle de vie uniquement pélagique. D'autres comme les tuniciers, organismes pélagiques en forme de tonneau, vont avoir un cycle de reproduction alternant phases sexuée hermaphrodite et asexuée.

- Justine Gadreaud - Comme détaillé par Guillaume ci-dessus, le cycle de vie des méduses, et en particulier ceux de la classe des scyphozoaires, inclut une variété de formes de développement : la larve planula, le polype, l'éphyrule, et enfin la méduse.

Toutes ces formes résultent de périodes de morphogenèse et de développement codées par un ensemble de gènes qui s'expriment en cascade. Nos connaissances académiques sur les gènes spécifiques de la morphogenèse chez les méduses sont récentes : le premier transcriptome complet des différents stades de développement d'Aurelia aurita a été réalisé pour la première fois en 2014 par Fuchs et al.*. Bien avant l'acquisition de ces données sur le détail des gènes codant pour la morphogenèse des différents stades de vie de la méduse, les éleveurs et scientifiques savaient que l'environnement influençait le déclenchement de ces changements de forme : variation de température et salinité de l'eau et quantité de nourriture disponible par exemple. L'épigénétique est la discipline de la biologie qui étudie la nature des mécanismes modifiant de manière réversible, transmissible et/ou adaptative l'expression des gènes sans en changer le code. De nombreuses pistes restent à explorer pour comprendre la reproduction et la morphogenèse des méduses.
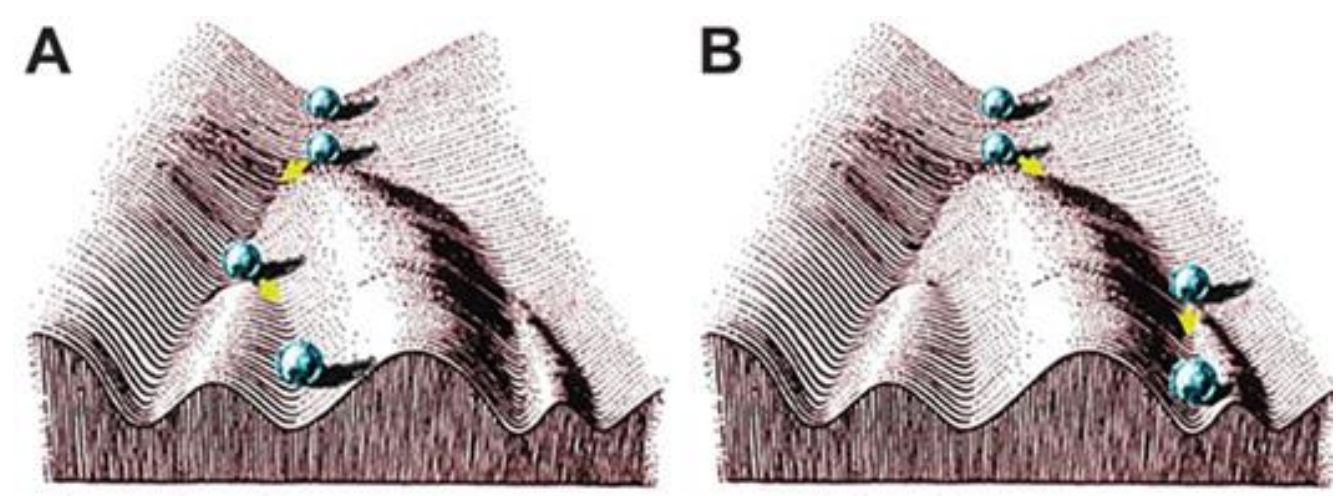

[5] Modèle de paysage épigénétique de Conrad Hal Waddington et destin des cellules 
- Shanta Rao - À propos de devenir, je tiens pour exemplaire la représentation par biologiste Waddington de son propre concept de paysage épigénétique évoquant la non-linéarité du programme, de la destinée auxquels les êtres vivants sont soumis. On y voit un ensemble de trajectoires possibles d'une cellule souche qui réaffirme la pluripotence du vivant et sa plasticité. Mais au-delà d'une lecture diachronique de la vie, tu évoquais, Justine, la géométrie variable de certaines méduses dans un temps parfois très court au sein même ton laboratoire, qu'en est-il ?

- Justine Gadreaud - La capacité de la méduse à changer de morphologie en fonction de la qualité de son environnement est aussi surprenante. Plusieurs observations personnelles ont montré qu'une méduse dans un espace confiné, avec peu de nourriture et sans les conditions physico-chimiques optimales à son développement et sa croissance, va énormément rétrécir, pouvant passer de $20 \mathrm{~cm}$ de diamètre à moins de $3 \mathrm{~cm}$ de diamètre en quelques jours mais survivre, tel un homme s'amaigrissant dans les mêmes conditions plusieurs semaines. Cette capacité s'ajoute à la fascination que m'évoque ces organismes, justement au regard de leurs 600 millions d'années d'existence, de leur morphologie si singulière dans le monde vivant et leur grande tolérance environnementale.

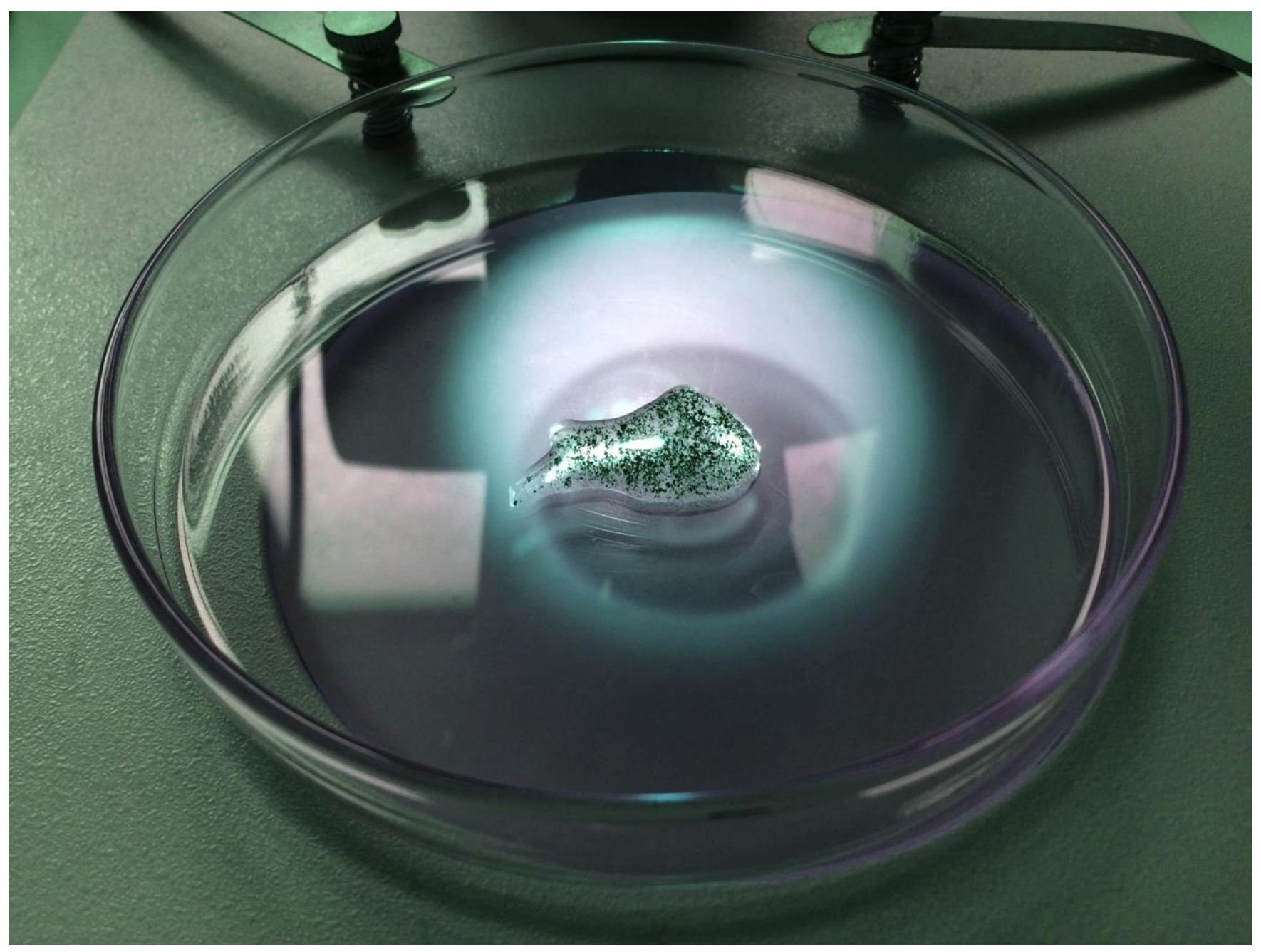

[6] Shanta Rao, Sans titre, 2018, tirage numérique, dimension variable, Polypes de méduse Aurelia sp. et son substrat Institut Méditerranéen de Biodiversité et d'Ecologie (IMBE), Marseille

- Shanta Rao - Né de nos discussions et d'expérimentations en atelier sur le site de la fondation Camargo, le corpus d'œuvres initié lors de la résidence Les Calanques, territoire de science et source d'inspiration puisent ses sources dans les singularités biologiques de certaines méduses : leur plasticité métabolique extrême qui leur permet des transformations allant de simples ajustements modifiant peu leur forme originelle (changement d'échelle, régénération de parties du corps) à des transformations radicales, de forme et même de matière ; leurs changements de territoires en fonction du stade de leur (C) 2020 ISTE OpenScience - Published by ISTE Ltd. London, UK - openscience.fr 
cycle de vie qui induit une dualité pélago-benthique ou pour le dire autrement, fluide-solide ; leur matière qui semble singulière à l'humain mais qui pourtant constitue plus de la moitié de la matière organique planétaire, une matière molle appartenant au champ des fluides complexes et qui se dérobe souvent à la vue ou lors de sa préhension ; leurs couleurs anisotropiques, variant selon la direction de leur perception.

Certains artistes ont commencé dès les années 1960 à utiliser des matériaux souples naturels ou synthétiques et ont donné naissance un nouveau champ de la sculpture, la Soft Sculpture. Ils ont ouvert ce champ d'expérimentation artistique notamment grâce aux nouveaux matériaux à structure moléculaire complexe issus de l'industrie pétrochimique mais il s'agissait avant tout pour eux de remettre en cause l'existence d'une force supérieure qui dominerait la matière (l'Homme, l'artiste, Dieu) et la pensée verticale d'une sculpture où les formes préexistent à leur incarnation matérielle. Ces artistes accueillent au contraire la plasticité inhérente aux matériaux qu'ils utilisent selon une approche en coalescence avec le monde physique dont nous faisons tous partie.

Les œuvres dont il est question dans cet article sont pour la plupart des soft sculptures qui ont la spécificité d'être constituées exclusivement de peinture. À l'image de l'ensemble de mes œuvres, elles se développent en plusieurs stades, dans un continuum de transformations qui témoigne d'un monde au devenir permanent.

Leur processus de fabrication s'apparente aux stades du cycle de vie de certaines méduses. Tout comme ces dernières, les œuvres ont un début d'existence lié à un substrat solide, une matrice en quelque sorte, sur laquelle la peinture sera appliquée par couches successives. Ces matrices, des fragments de carcasses automobiles, évoquent la présence grandissante en milieu marin d'éléments solides d'origine humaine responsables de l'accroissement de la population des méduses.

Tout comme le collagène planctonique, la peinture utilisée pour la réalisation des œuvres est un polymère appartenant au champ des fluides complexes et présentant une grande variété de comportements entre les états solide et liquide. Les pigments employés possèdent, quant à eux, des propriétés optiques semblables aux couleurs opalescentes du monde marin. Techniquement empreinte, elle sera ensuite décollée de sa matrice et libérée de son référent : l'œuvre débute son existence de soft sculpture pour être activée au sein d'expositions.

L'une des premières soft sculptures a été présentée lors de l'exposition Les Calanques, territoire de science et source d'inspiration au Fonds Régional d'Art Contemporain Provence-Alpes-Côte-d'Azur de Marseille en 2018, dans le cadre d'une proposition installative dont le titre, La couleur tombée du ciel, est emprunté à une œuvre de H.P. Lovecraft. Elle y était exposée dans une phase évolutive légèrement décollée de sa matrice encore présente -, sur un lit de drapés iridescents évoquant les interactions mer-atmosphère. Se donnaient également à voir un aquarium-boule ainsi qu'un diaporama diffusé en un mouvement ondulatoire sur un moniteur ; paysages marins, machines cinétiques simulant le mouvement perpétuel des courants méditerranéens, expérimentations plastiques, station d'étude de la mécanique des fluides océaniques, organismes ou œuvres en gestation, ces images témoignent autant de la connexité des hommes avec la nature que de celle avec les dispositifs expérimentaux et machines qui leur permettent de prolonger, au sein des microcosmes artificiels que sont les laboratoires scientifiques et ateliers d'artistes, les mouvements de sa matière organique ou inorganique. Guillaume, tu disais des machines d'expérimentation scientifique qu'elles étaient des porte-paroles? 


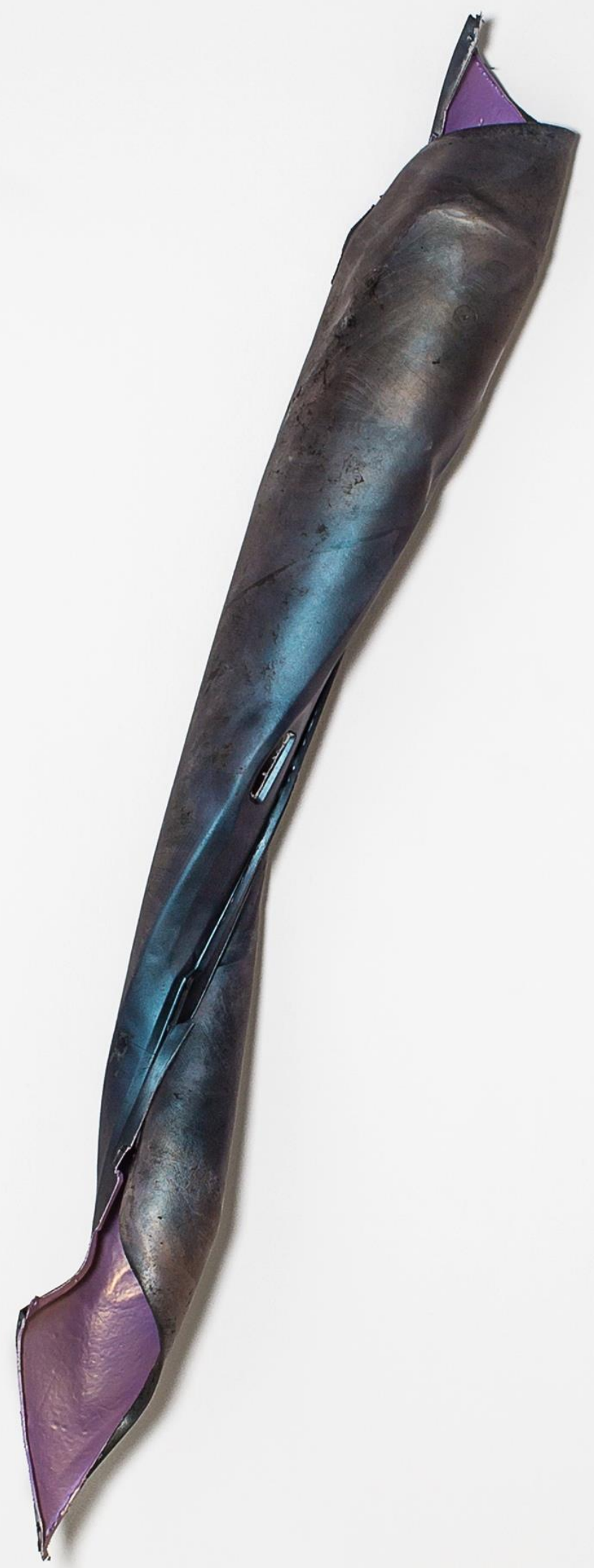

[7] Shanta Rao, Sans titre, 2018, peinture polymère, $200 \times 20 \times 17 \mathrm{~cm}$ Exposition PULPE, en duo avec Mimosa Echard, commissariat Raphaël Brunel Galerie Edouard Manet, Gennevilliers 
- Guillaume Marchessaux - Dans notre domaine de recherche, l'océanographie, nous effectuons différentes mesures. Dans un premier temps, notre démarche consiste à se poser une question sur un phénomène observé, par exemple comment les proliférations de méduses sont provoquées et quels sont les impacts socio-écologiques? Puis, nous nous rendons sur le terrain et nous prélevons et mesurons les paramètres chimiques, physiques, biologiques et écologiques à l'aide de sondes, de bouteilles et de filets. Dans mon cas, il s'agit de comprendre la dynamique temporelle du zooplancton, des méduses et des autres espèces gélatineuses en lien avec les paramètres environnementaux.

Comme nous ne pouvons être à temps plein sur le terrain, nous recréons les conditions environnementales en laboratoire. Cela nous permet de moduler les paramètres (température, salinité, quantité de nourriture, etc.) comme nous le souhaitons et quantifier les taux physiologiques des espèces modèles étudiées. A partir de cela nous pouvons déterminer les paramètres optimums pour le développement de l'espèce modèle étudiée et ainsi coupler ces mesures en laboratoire à celles récoltées sur le terrain.

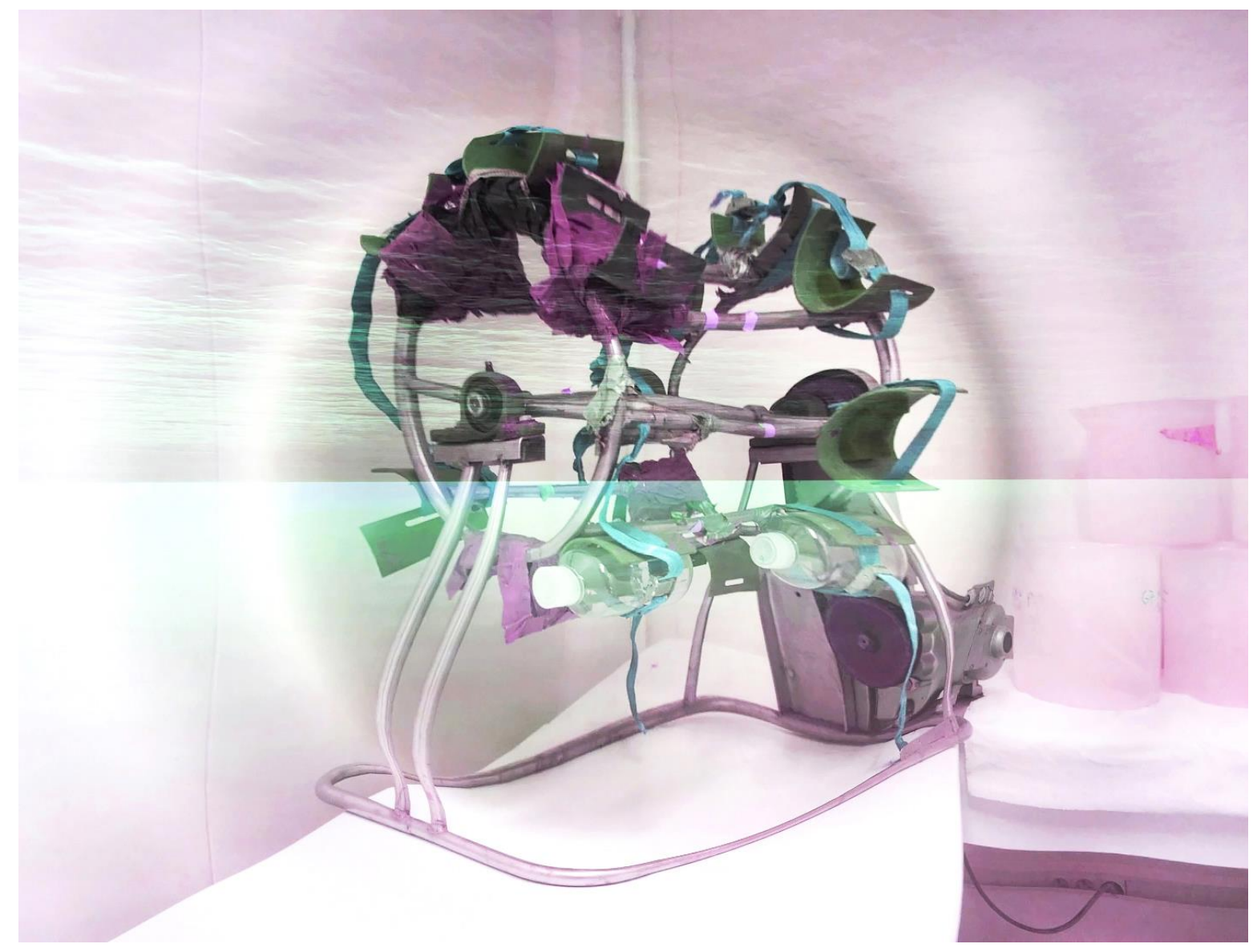

[8] Shanta Rao, La couleur tombée du ciel, 2018, diaporama

Exposition Les Calanques, territoire de sciences, source d'inspiration

Fonds Régional d'Art Contemporain Provence-Alpes-Côte-d'Azur, Marseille/FR, 2018

D'après une photographie de machine cinétique réalisée dans le laboratoire de Guillaume Marchessaux (Institut Méditerranéen d'Océanologie, Marseille)

Le plancton est défini comme l'ensemble des organismes qui errent aux grés des courants. Ces organismes sont incapables de se maintenir seuls en suspension dans la colonne d'eau, ils sont dépendants des mouvements d'eau, les courants. Dans le cas de l'étude du plancton en laboratoire, nous avons recourt à un certain nombre de machines cinétiques. Ces machines ont pour principale mission de créer artificiellement un faible mouvement d'eau permettant le maintien du plancton et des méduses en suspension durant les expériences. Ces machines font partie intégrale de mon travail 
quotidien sur le plancton. Ces machines, anthropiques et artificielles, sont finalement des porte-paroles des processus naturels que l'on cherche à montrer.

- Shanta Rao - Vous disiez de notre collaboration artiste-chercheurs qu'elle avait renouvelé le regard et la fascination que vous portiez à ces organismes, qu'une lecture à travers le prisme de l'art avait accru le temps dédié à leur contemplation et aussi ouvert des champs de réflexion scientifique que vous n'aviez jusqu'alors pas ou peu explorés comme les questions d'origine ou de morphologie. Et qu'au final, démarche scientifique et artistique étaient souvent très proches. Cette collaboration a, pour ma part, permis l'approche de modèles affranchis de toute idée de finitude, formelle mais aussi dans la définition même de la vie - est vivant ce qui nait et meurt - ici remise en cause par l'immortalité biologique de certaines méduses. C'était aussi une invitation à la pensée d'un monde plus fluide et coalescent dans lequel il serait possible de repenser les relations qui nous unissent tant à l'ensemble du vivant que du non vivant. Quel serait le langage du futur si l'abandon de la maîtrise (de l'illusion de la maîtrise) nous amenait à entreprendre un dialogue et non une guerre avec ce qui nous entoure et que, bizarrement, nous appelons «environnement » ? écrivait le paysagiste Gilles Clément ${ }^{1}$.

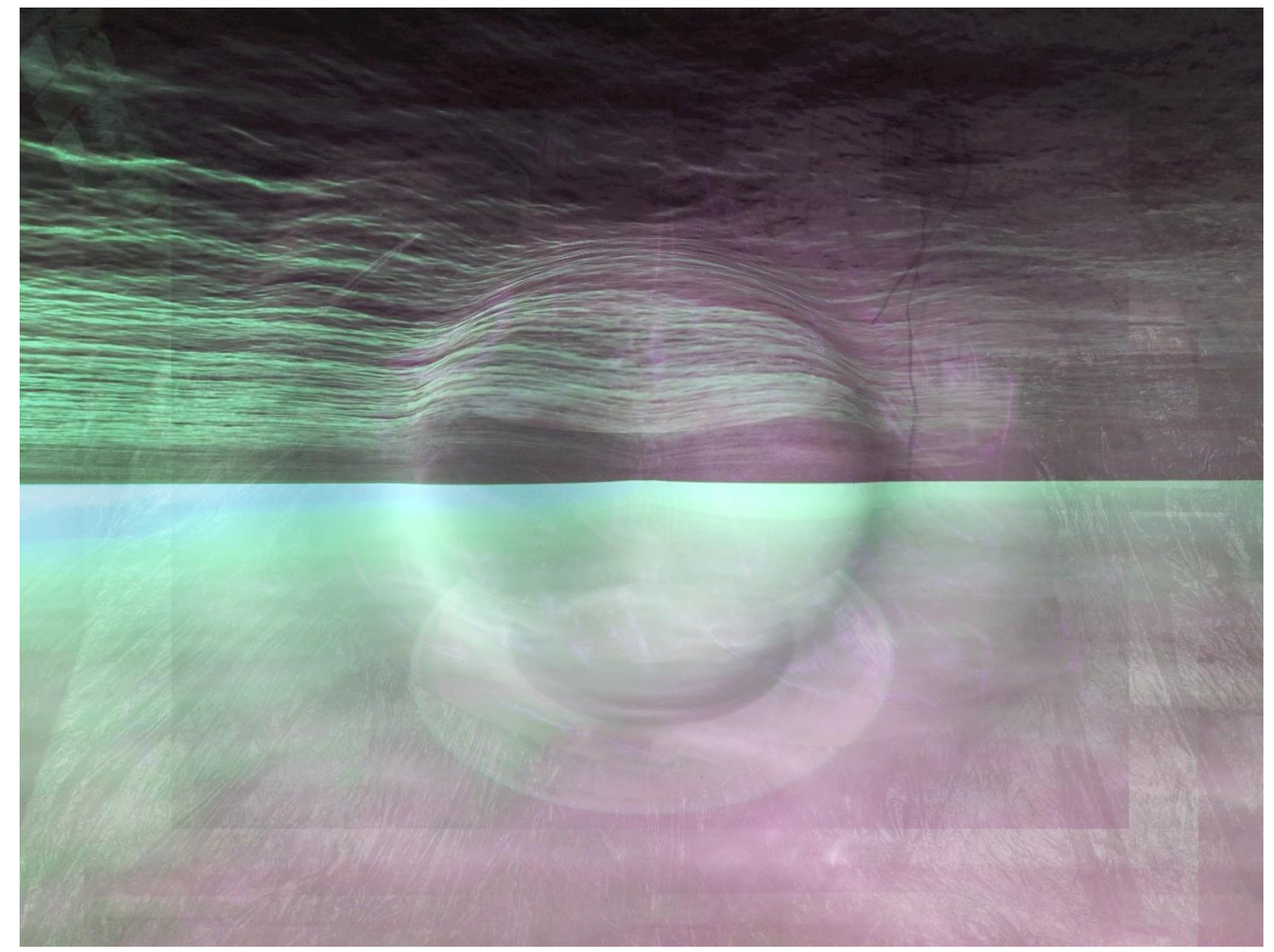

[9] Shanta Rao, La couleur tombée du ciel, 2018, capture vidéo

Exposition Les Calanques, territoire de sciences, source d'inspiration, commissariat David Moinard Fonds Régional d'Art Contemporain Provence-Alpes-Côte-d'Azur, Marseille

D'après une photographie d'aquarium-boule réalisée dans le laboratoire de Justine Gadreaud (Institut Méditerranéen de Biodiversité et d'Ecologie, Marseille)

\footnotetext{
${ }^{1}$ texte d'ouverture du programme de résidence Les Calanques, territoire de science et source d'inspiration, 2017.
} 


\section{Remerciements}

Cette collaboration artiste-chercheurs n'aurait pas été possible sans le soutien de nombreuses personnes. Nous remercions la Fondation Camargo, l'OSU Pythéas, le Parc national de Calanques et Gilles Clément de nous avoir accueillis et soutenus dans le cadre de la résidence artistique Les Calanques, territoire de science et source d'inspiration. Nous remercions également les laboratoires Institut Méditerranéen d'Océanologie (MIO) et l'Institut Méditerranéen de Biodiversité et d'Ecologie (IMBE) de nous avoir accueillis. Un grand merci à Jean Blanchot (MIO) pour ses échanges avec Shanta Rao et à Guillemette Caulliez (LASIF) de nous avoir accueillis et montré la Grande Soufflerie Air-Eau de Luminy. Merci également à Guillaume Letestu, pêcheur professionnel à Cassis d'avoir permis à Shanta Rao de sortir en mer. Merci à Giles Dyfresne (Dynamic Auto, Carnoux-en-Provence) et Barnabé Morreton (Casse Marine Méditerranée, Carnoux-en-Provence) d'avoir fourni des éléments de carrosserie à Shanta Rao pour la création de ses œuvres. Enfin, merci à Anne Prouha, Nicolas Garnier et Cristel Sevilla (Académie Aix-Marseille) de nous avoir accueillis à l'école élémentaire " Les abeilles » (La Ciotat) dans laquelle nous avons pu présenter nos travaux à des élèves.

\section{Références}

FUCHS B., WANG W., GRASPEUNTNER S., LI Y., INSUA S., HERBST E.-M., DIRKSEN P., BÖHM A.-M., HEMMRICH G., SOMMER F., « Regulation of Polyp-to-Jellyfish Transition in Aurelia aurita », Current Biology, n 24(3), p. 263-273, 2014.

\section{Crédits photographiques}

1.2.3.6.8.9 @ Shanta Rao

4. (C) Justine Gadreaud

7. (C) Margot Montigny - Emba /galerie Edouard Manet, Gennevilliers, 2019

\section{Plus d'informations}

Site web de Shanta Rao | http://shantarao.net/

Site web de Guillaume Marchessaux | https://guillaume-marchessaux-94.webself.net/

À propos du programme de résidence Les Calanques, territoire de science et source d'inspiration https://camargofoundation.org/fr/programmes/les-calanques/ 